\title{
Caracterização físico-química e desenvolvimento pós-colheita de jabuticabas (Plinia peruviana e P. cauliflora)
}

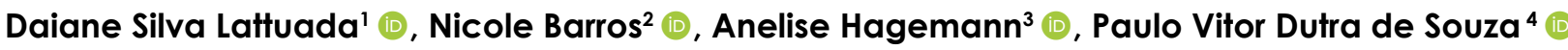 \\ ' Secretaria da Agricultura, Pecuária e Desenvolvimento Rural do Estado do Rio Grande do Sul, Departamento de Diagnóstico \\ e Pesquisa Agropecuária, Rua Gonçalves Dias, 570, CEP 90130-060, Porto Alegre, Rio Grande do Sul, Brasil \\ *Autor para correspondência: daiane-lattuada@agricultura.rs.gov.br \\ 2 Universidade Federal do Rio Grande do Sul, Rua Ramiro Barcelos, 2400, CEP 90035-003, Porto Alegre, Rio Grande do Sul, Brasil \\ ${ }^{3}$ Secretaria da Agricultura, Pecuária e Desenvolvimento Rural do Estado do Rio Grande do Sul, Departamento de Infraestrutura Rural, \\ Irrigação e Usos Múltiplos de Água, Avenida Celina Chaves Kroeff, portão 10, Esteio, Rio Grande do Sul, Brasil \\ ${ }^{4}$ Universidade Federal do Rio Grande do Sul, Faculdade de Agronomia, Departamento de Horticultura e Silvicultura \\ Av. Bento Gonçalves, 7712, CEP 91540-000, Porto Alegre, Rio Grande do Sul, Brasil
}

Recebido em 19.111.2019

Aceito em 15.VI.2020

DOI 10.21826/2446-82312020v75e2020015

RESUMO - Plinia peruviana e P. cauliflora pertencem à família Myrtaceae, apresentam potencial para exploração como frutífera e/ou para arborização urbana. Contudo, pouco se conhece sobre suas características, sendo o objetivo deste trabalho avaliar morfometria (dimensão, massa fresca de frutos, polpa, casca e sementes, rendimento de polpa, número de sementes por fruto e cor dos frutos), constituição química (sólidos solúveis SS, acidez titulável AT, Ratio e teor vitamina C) e desenvolvimento pós-colheita (constituição química e perda de massa fresca) dos frutos destas espécies. As amostras estudadas são arredondadas, com alto teor de água ( $83 \%$ ), alto rendimento de polpa (entre 67 e $76 \%$ ), massa fresca entre 5,30 e 6,82 g e mais de uma semente por fruto. Apresentaram entre 11,4 e $12,7 \mathrm{SS}$, baixa AT e $19 \mathrm{mg} 100 \mathrm{~g}_{\text {de polpa }}^{-1}$ de vitamina C. Plinia peruviana e P. cauliflora apresentam características físico-químicas similares, podendo ser armazenadas por 28 dias sob-refrigeração $\left(\approx 2^{\circ} \mathrm{C}\right)$.

Palavras-chave: frutífera nativa, morfologia, Myrtaceae, química dos frutos

ABSTRACT - Characterization and post-harvest behavior of jabuticabas Plinia peruviana and $P$. cauliflora. Plinia peruviana and $P$. cauliflora are Myrtaceae with potential for agricultural exploitation or urban afforestation. However, there is not much information about its characteristics; consequently, making the objective of this work to evaluate morphometry (size, fresh fruit mass, pulp, bark and seeds, pulp yield, number of seeds per fruit and fruit color), chemical composition (soluble solids, acid titratable AT, Ratio and vitamin C content) and post-harvest behavior (chemical composition and loss of fresh mass) of its fruits. The samples studied were rounded, with high water content (83\%), high yield of pulp (between 67 and 76\%), fresh mass between 5.30 and $6.82 \mathrm{~g}$ and more than one seed per fruit. They presented between 11.4 and $12.7 \mathrm{SS}$, low AT and $19 \mathrm{mg}$ $100 \mathrm{~g}$ of pulp- 1 of vitamin C. Plinia peruviana and P. cauliflora have shown similar physicochemical characteristics and can be stored for 28 days under refrigeration $\left(\approx 2^{\circ} \mathrm{C}\right)$.

Key-words: chemical of fruits, Myrtaceae, morphology, native fruit tree

\section{INTRODUÇÃO}

As jabuticabeiras, pertencentes a Myrtaceae, são arbustos ou árvores, nativas do Brasil estando atualmente todas espécies alocadas no gênero Plinia. No país são citadas 33 espécies e destas, 28 são endêmicas (Flora do Brasil 2020). Podem ser encontradas nas cinco regiões do país, mas é nos Estados de Goiás, São Paulo e Minas Gerais, respectivamente, que ocorrem as maiores produções comerciais (IBGE 2017). Nove espécies são conhecidas, entre as quais se destacam Plinia trunciflora Berg, conhecida como jabuticaba de cabinho; Plinia cauliflora (DC) Berg, como jabuticaba paulista ou jabuticaba-açu, e Plinia jaboticaba (Vell.) Berg, como jabuticaba Sabará, sendo esta a mais comercializada no mercado brasileiro (Danner et al. 2011). Ainda há Plinia peruviana (Poir.)
Govaerts que pode ser encontrada nas regiões sul e sudeste (Flora do Brasil 2020).

$\mathrm{O}$ fruto das jabuticabeiras cresce diretamente do tronco principal e ramos, apresenta de 3-4 cm de diâmetro, possui epiderme roxa, polpa branca mucilaginosa e agridoce, muito saborosa, podendo apresentar até quatro sementes. O sabor doce e sub-ácido da fruta deve-se provavelmente ao seu conteúdo de açúcares, ácidos orgânicos e conteúdo de terpenos (Plageman et al. 2012).

A potencialidade econômica desta frutífera abrange o mercado de frutos in natura ou industrializados na forma de bebidas, geleias, doces, sorvetes, picolés, entre outras formas de uso (Sasso et al. 2010); contudo os frutos são altamente perecíveis, apresentando curto período de utilização, devido ao seu elevado teor de água e açúcares. Depois de colhido, o fruto apresenta 
vida útil de aproximadamente três dias, o que prejudica sua comercialização (Silva et al. 2017). Contudo, esse problema não tem recebido a atenção necessária, já que há um desconhecimento das características físicas e químicas dos frutos em função do local de cultivo, tanto no que se refere à fase de produção, quanto à fase de pós-colheita (Oliveira et al. 2003). Sendo que estas características podem variar em função do cultivar, condições climáticas, locais de cultivo, manejo e tratamentos fitossanitários (Chitarra \& Chitarra 2005).

Nos últimos anos, tem aumentado a demanda mundial por novos produtos que conciliem geração de renda com conservação do meio ambiente (Ronchi 2017). As pessoas, ainda que timidamente, tem se preocupado com o uso da biodiversidade de maneira consciente e tem incentivado a compra de produtos de empresas que seguem esse quesito. Neste contexto verifica-se o interesse crescente por espécies de frutíferas nativas, o que incentiva o desenvolvimento de pesquisas sobre as suas características nutricionais, qualidade dos frutos e métodos de conservação.

A qualidade dos frutos corresponde ao conjunto de atributos ou propriedades que os tornam apreciados como alimentos, dos quais dizem respeito a aparência, o rendimento de polpa, o número de sementes, o sabor, o aroma, a textura e o valor nutritivo. O conhecimento destes atributos assume grande importância, uma vez que podem ser utilizadas técnicas para a sua preservação e seleção de variedades. Algumas análises químicas, como sólidos solúveis (SS), pH, acidez titulável (AT) e vitamina $\mathrm{C}$, fornecem informações importantes para caracterizar a qualidade dos frutos para consumo.

Como técnicas pós-colheita, a utilização do armazenamento refrigerado e de embalagens, estão entre os meios mais eficazes na conservação de produtos in natura. Essas técnicas são efetivas, pois retardam os processos metabólicos envolvidos na ação das enzimas degradativas e oxidativas, reduzem a respiração e a produção do etileno; assim como retardam o crescimento dos microorganismos, sendo a eficiência de controle maior quanto mais rápido se processa (Silveira et al. 2005). A qualidade pós-colheita dos frutos está relacionada com a minimização da taxa de deterioração, ou seja, manutenção da firmeza, cor, aparência, visando mantê-los atraentes ao consumidor por um período de tempo mais longo (Chitarra \& Chitarra 2005).

Portanto, estudos sobre a caracterização de frutos de diferentes amostras e o comportamento pós-colheita são necessários para expandir o conhecimento desta cultura, à fim de que se possam identificar indivíduos com características agronômicas desejáveis e futuramente adotar métodos para conservação dos frutos sem afetar a qualidade das jabuticabas.

O objetivo do trabalho foi caracterizar físicoquimicamente frutos de amostras de Plinia peruviana e de $P$. cauliflora e determinar sua longevidade pós-colheita.

\section{MATERIAL E MÉTODOS}

O material vegetal utilizado foi colhido de cinco árvores de um pomar de jabuticabeiras encontrado na região metropolitana de Porto Alegre (30 5'43"S; 51 '9'11'O, altitude $117 \mathrm{~m}$ ). As árvores selecionadas para o estudo foram numeradas (de dois a seis) e esta identificação foi mantida para determinar as amostras.

Frutos inteiros e maduros de três amostras de $P$. peruviana e dois de $P$. cauliflora foram colhidos manualmente (coleta única), e armazenados em sacos de polietileno para transporte até o laboratório, onde foram feitas as análises de caracterização físico-química dos frutos e desenvolvimento pós-colheita. A primeira análise ocorreu 24 horas após a colheita e, o acompanhamento para verificar o desenvolvimento pós-colheita ocorreu semanalmente, durante 28 dias (totalizando cinco avaliações). Para isto, os frutos permaneceram armazenados em embalagens plásticas (Nova Pack ${ }^{\circledR}$, NP-10 com dimensões internas: $159 \times 80 \times 56 \mathrm{~mm})$, sob-refrigeração $\left(\approx 2{ }^{\circ} \mathrm{C}\right)$.

$\mathrm{Na}$ caracterização físico-química, para todos os parâmetros analisados foram utilizados quatro repetições de 15 frutos, por amostra. Os frutos foram descritos considerando-se os seguintes aspectos: dimensões (diâmetro transversal, longitudinal e a relação entre eles, expresso em $\mathrm{mm}$ ), número de sementes por fruto, massa fresca de frutos, de cascas e de sementes $(\mathrm{g})$, rendimento de polpa (razão entre a massa dos frutos e das cascas e sementes, expresso em \%). Utilizou-se, para a avaliação das dimensões dos frutos, paquímetro digital com precisão de $\pm 0,2 \mathrm{~mm}$ e balança analítica digital para as análises de massa. Ainda, foi avaliado, no momento da colheita, o teor de água dos frutos (\%) por diferença de massa; para isso, três repetições de cinco frutos foram pesados e secos em estufa $\left(65^{\circ} \mathrm{C}\right)$ até peso constante.

Mediu-se também a coloração do epicarpo mediante uso de colorímetro, procedendo-se duas medições equidistantes no fruto, onde foram verificados os índices luminosidade $\left(\mathrm{L}^{*}\right)$, o espectro vermelho-verde $\left(\mathrm{a}^{*}\right)$ e o espectro amareloazul $\left(\mathrm{b}^{*}\right) \mathrm{e}$, a partir destes, calculado o ângulo hue $\left({ }^{\circ} \mathrm{Hue}=\right.$ $\left.\tan -1 b^{*} / a^{*}\right)$, que representa as nuances de coloração e a cromaticidade que representa a intensidade/brilho.

Para as análises de constituição química, a polpa dos frutos, separada manualmente de cascas e sementes, foi triturada e homogeneizada integralmente em multiprocessador doméstico. As análises em triplicata de sólidos solúveis (SS), acidez titulável (AT), Ratio (SS/AT) e teor de vitamina C (expresso em mg 100g de polpa $^{-1}$ de ácido ascórbico), foram feitas de acordo com metodologia da AOAC (1997), sendo os sólidos solúveis medidos, imediatamente após a trituração, através do uso de refratômetro digital. Para avaliação da acidez titulável e teor de vitamina $\mathrm{C}$ as amostras foram armazenadas em ultrafreezer $\left(\approx-50^{\circ} \mathrm{C}\right)$, até o momento da análise.

Para verificar o desenvolvimento pós-colheita foram repetidos, semanalmente, os mesmos procedimentos para acidez titulável, sólidos solúveis e teor de vitamina $\mathrm{C}$ e, 
ainda determinada a perda de massa, por diferença de massa fresca, ao longo do período de armazenamento (\%).

$\mathrm{O}$ delineamento experimental adotado foi o completamente casualizado com quatro repetições de quinze frutos por amostra, sendo as amostras consideradas como tratamentos. Para comparação entre as espécies vegetais utilizou-se o teste de comparação de médias LSD $(\mathrm{p}<0,001)$. Para comparação entre as amostras, os dados de caracterização físico-química dos frutos foram submetidos à análise de variância $(\mathrm{p}<0,01)$, sendo utilizado o teste de comparação múltipla de médias de Duncan $(\mathrm{p}<0,05)$, quando houve diferença significativa entre os tratamentos. Para a avaliação do comportamento pós-colheita utilizouse análise de regressão.

\section{RESULTADOS E DISCUSSÃO}

Entre as duas espécies estudadas somente foram observadas diferenças significativas através do teste de comparação de média LSD $(\mathrm{p}<0,001)$, para os parâmetros de cor L*, a* e b*, onde $P$. cauliflora foi superior à $P$. peruviana e para os parâmetros de análise física onde $P$. peruviana superou $P$. cauliflora em diâmetro transversal e longitudinal dos frutos e massa fresca de frutos e sementes. Portanto, P. cauliflora apresenta maior luminosidade e tons mais pronunciados de vermelho e amarelo, comparado à $P$. peruviana. Ainda, P. peruviana, produz frutos de maiores dimensões. Nas demais variáveis avaliadas não foram encontradas diferenças significativas entre as espécies. Logo, P. peruviana apresenta características físico-químicas similares à $P$. cauliflora, podendo ter o mesmo potencial de utilização (Tabs. 1 - 4).

$\mathrm{Na}$ comparação entre as amostras, os frutos das duas espécies de jabuticabeira apresentaram grande variabilidade, tanto para as características físicas, quanto para as químicas (Tabs. 1 - 3).

Maior diâmetro longitudinal foi observado na amostra 6 com 22,45 mm, porém não diferiu das amostras 3 e
4 (21,67 e 21,38 mm, respectivamente - Tab. 1). Os menores diâmetros foram obtidos nas amostras 2 e 5 . Para diâmetro transversal, a amostra 6 também superou as demais amostras (com 22,26 mm), embora não tenha diferido da amostra $3(21,55 \mathrm{~mm})$ (Tab. 1). As demais amostras apresentaram diâmetros transversais semelhantes entre si. Os resultados da relação entre os diâmetros transversal e longitudinal indicam que os frutos são arredondados, não havendo diferença significativa entre as amostras (Tab. 1).

A massa fresca dos frutos variou entre 5,30 e 6,82 g, sendo a amostra 6 significativamente superior as demais em massa, tanto de frutos como também de cascas e sementes, embora para este último parâmetro não tenha diferido das amostras 3, 4 (Tab. 2).

Não foram observadas diferenças significativas quanto ao rendimento de polpa entre as amostras (Tab. 2). Contudo, o rendimento de polpa encontrado neste estudo foi praticamente duas vezes ao encontrado por Boari Lima et al. (2009), com jabuticabeira Paulista (49\%) e jabuticabeira Sabará (36\%), (P. cauliflora e P. jaboticaba, respectivamente). Rendimento de polpa é um parâmetro importante a ser avaliado pelas indústrias de processamento de frutos

Zerbielli et al. (2016), estudando a diversidade físicoquímica dos frutos de 40 genótipos de jabuticabeira ( $P$. cauliflora) de ocorrência natural, no município de Passo Fundo (RS), encontraram massa fresca de frutos variando entre 6,4 e $11,4 \mathrm{~g}$, massa fresca de sementes entre 0,11 e $0,36 \mathrm{~g}$ e percentual de polpa entre 57,8 e $76,1 \%$. Comparativamente, as amostras estudadas pelos autores acima eram de maior massa fresca de frutos e de menor massa fresca de sementes que as encontradas no presente estudo. No entanto, o rendimento de polpa de todas as amostras deste trabalho enquadram-se entre os de maior rendimento encontrados por Zerbielli et al. (2016). Neste sentido, P. peruviana e P. cauliflora podem ser mais promissoras para o processamento em agroindústrias, por apresentarem melhor aproveitamento de frutos.

Tabela 1. Diâmetro transversal e longitudinal e a relação entre os diâmetros transversal e longitudinal de frutos de cinco amostras de jabuticabeira coletadas em Porto Alegre.

\begin{tabular}{|c|c|c|c|}
\hline $\begin{array}{l}\text { Comparação entre: } \\
\text { Amostras/indivíduos }\end{array}$ & Diâmetro Transversal (mm) & Diâmetro Longitudinal (mm) & Relação Transversal/Longitudinal \\
\hline P. peruviana (3) & $21,55 \mathrm{ab}$ & $21,67 \quad a b$ & 0,99 \\
\hline P. peruviana (4) & $21,15 \quad b$ & $21,38 \quad a b$ & 0,98 \\
\hline P. peruviana (6) & 22,26 a & 22,45 a & 0,99 \\
\hline P. cauliflora (2) & $20,72 \quad b$ & $20,67 \quad b$ & 1,00 \\
\hline P. cauliflora (5) & $20,72 \quad b$ & $20,69 \mathrm{~b}$ & 1,00 \\
\hline \multicolumn{4}{|l|}{ Espécies } \\
\hline P. peruviana & 20,83 a & 20,85 a & 1,00 \\
\hline P. cauliflora & $20,01 \quad \mathrm{~b}$ & $19,88 \quad \mathrm{~b}$ & 0,99 \\
\hline CV \% Amostras & 2,80 & 3,17 & $1,12(\mathrm{~ns})$ \\
\hline CV \% Espécies & $10,76 \quad * *$ & $10,51 \quad * * *$ & $3,38(\mathrm{~ns})$ \\
\hline
\end{tabular}

Médias seguidas de mesma letra, na coluna, não diferem entre si pelo teste de Duncan $(p<0,05)$, entre as amostras e LSD ( $<<0,001)$ entre as espécies, ns: Variância não significativa. 
Tabela 2. Massa fresca de frutos, cascas e sementes $(\mathrm{g})$; rendimento de polpa (\%); teor de água dos frutos e número de sementes por fruto de cinco amostras de jabuticabeira coletados em Porto Alegre.

\begin{tabular}{|c|c|c|c|c|c|c|}
\hline $\begin{array}{l}\text { Comparação entre: } \\
\text { Amostras/indivíduos }\end{array}$ & $\begin{array}{c}\text { Massa Fresca de } \\
\text { Frutos }(\mathrm{g})\end{array}$ & $\begin{array}{c}\text { Massa Fresca de } \\
\text { cascas }(\mathrm{g})\end{array}$ & $\begin{array}{c}\text { Massa Fresca de } \\
\text { Sementes }(\mathrm{g})\end{array}$ & $\begin{array}{l}\text { Rendimento } \\
\text { de polpa }(\%)\end{array}$ & $\begin{array}{c}\text { Teor de água } \\
\text { dos Frutos }(\%)\end{array}$ & $\begin{array}{c}\text { Número de } \\
\text { Sementes/Fruto }\end{array}$ \\
\hline P. peruviana (3) & $6,04 \mathrm{~b}$ & $1,43 \mathrm{c}$ & $0,20 \mathrm{a}$ & 72,04 & 83,48 & 1,33 \\
\hline P. peruviana (4) & $5,77 \mathrm{~b}$ & $1,72 \mathrm{~b}$ & $0,20 \mathrm{a}$ & 71,44 & 83,18 & 1,28 \\
\hline P. peruviana (6) & $6,82 \mathrm{a}$ & $1,98 \mathrm{a}$ & 0,19 a & 68,08 & 83,33 & 1,25 \\
\hline P. cauliflora (2) & $5,30 \mathrm{~b}$ & $1,51 \mathrm{bc}$ & $0,15 \mathrm{~b}$ & 73,03 & 82,55 & 1,28 \\
\hline P. cauliflora (5) & $5,43 \mathrm{~b}$ & $1,51 \mathrm{c}$ & $0,15 \mathrm{~b}$ & 69,70 & 84,23 & 1,16 \\
\hline \multicolumn{7}{|l|}{ Espécies } \\
\hline$P$. peruviana & 6,21 a & 1,71 & $0,20 \mathrm{a}$ & 70,65 & 16,66 & 1,28 \\
\hline P. cauliflora & $5,37 \mathrm{~b}$ & 1,51 & $0,15 \mathrm{~b}$ & 71,37 & 16,60 & 1,22 \\
\hline CV \% Amostras & 8,38 & 8,46 & 12,19 & $3,55(\mathrm{~ns})$ & $2,35(\mathrm{~ns})$ & $13,15(\mathrm{~ns})$ \\
\hline CV \% Espécies & $9,85 * *$ & 13,62 (ns) & $11,24 * * *$ & $4,29(\mathrm{~ns})$ & $10,90(\mathrm{~ns})$ & $12,59(\mathrm{~ns})$ \\
\hline
\end{tabular}

Médias seguidas de mesma letra, na coluna, não diferem entre si pelo teste de Duncan $(\mathrm{p}<0,05)$, entre as amostras e LSD ( $\mathrm{p}<0,001)$ entre as espécies, ns =variância não significativa.

O teor de água e o número de sementes por fruto não diferiram significativamente entre as amostras estudadas (Tab. 2). Os teores de água dos frutos observados estão de acordo com o estipulado pela Tabela Brasileira de Composição de Alimentos (Taco, 2006) para jabuticabas e similares aos encontrados para frutos inteiros de jabuticaba Paulista ( $P$. cauliflora, 80,35\%) e jabuticaba Sabará ( $P$. jaboticaba, 79,41\%) (Boari Lima et al. 2008). Os mesmos autores também observaram alto conteúdo de água nas frações do fruto (polpa, casca e sementes).

Sólidos solúveis, representado pela escala ${ }^{\circ}$ Brix, correspondem ao total de todos os sólidos dissolvidos na água, constituindo-se, basicamente, de açúcares (sacarose, frutose e glicose), sendo expresso, basicamente, pela percentagem de açúcar presente no alimento (Martins 2007). A concentração desses sólidos constitui-se em uma das variáveis mais importantes para medir a qualidade de frutos. As maiores quantidades de SS foram observadas nas amostras 5 e $6\left(12,4\right.$ e $12,75^{\circ}$ Brix, respectivamente $)$ (Tab. 3). A amostra 4 apresentou o menor teor de sólidos solúveis - SS (11,4 ${ }^{\circ}$ Brix) enquanto as amostras 2 e 3 apresentaram valores intermediários de SS. Boari Lima et al. (2008) observaram maiores valores de sólidos solúveis, comparados aos deste estudo, em polpa de jabuticaba Paulista (P. cauliflora) e Sabará (P. jaboticaba), com 14,90 e $14,30^{\circ} \mathrm{Brix}$, respectivamente, provavelmente em função das distintas condições de clima e solo. Por outro lado, os valores aqui observados foram intermediários aos obtidos no estudo de Zerbielli et al. (2016) com 40 genótipos de jabuticabeiras (P. cauliflora), onde observaram entre 10,8 e $15,8^{\circ}$ Brix.

Segundo Negreiros et al. (2008), a acidez do fruto é uma característica importante para a indústria, pois desfavorece a manifestação de microrganismos e, consequentemente, confere maior tempo de conservação do produto. Por outro lado, a acidez proporciona um sabor não muito doce, o que não agrada aos consumidores de frutos in natura. As amostras 2 e 4 se destacaram das demais quanto à acidez titulável $(0,83 \% ; 0,82 \%$ de ácido cítrico, respectivamente) (Tab. 3). A amostra 6 apresentou a menor acidez titulável $(0,69 \%)$ e as amostras 3 e 5 , mostraram índices intermediários. Estes valores de acidez foram menores aos observados em jabuticaba Paulista $(P$. cauliflora) e Sabará (P. jaboticaba) por Boari Lima et al. (2008), contudo foram muito superiores aos observados por Zerbielli et al. (2016), em 40 genótipos de jabuticabeira (média de 0,59\%).

A relação entre sólidos solúveis e acidez titulável (SS/ AT) é considerada um critério de avaliação do flavor em frutos além de indicativo do grau de maturação Chitarra \& Chitarra, 2005). A amostra 6 apresentou a maior relação entre sólidos solúveis e acidez titulável com 17,10 , enquanto que a amostra 2 , a menor relação $(13,12)$ (Tab. 3). As demais apresentaram ratio intermediários aos anteriores. Para Zerbielli et al. (2016), os valores de ratio variaram entre 36,5 a 42,5, devido principalmente à baixa concentração de acidez nas amostras estudadas. Danner et al. (2011) obtiveram mais ampla variação na relação SS/AT $(10,3$ a 63,2$)$ em jabuticabas provenientes de 36 genótipos (P. cauliflora) localizados em cinco municípios da região sudoeste do Paraná. Os valores observados no presente estudo foram muito inferiores aos demais, devido especialmente aos elevados índices de acidez titulável. A quantificação da relação entre o teor de sólidos solúveis totais e a acidez titulável está relacionada com o balanço entre açúcares e ácidos presentes na fruta, sendo importante indicativo do sabor (Kluge et al. 2002), determinando, pelo equilíbrio gustativo, a qualidade geral de um produto e a longevidade pós-colheita.

A vitamina $C$ não é sintetizada pelo organismo humano, o que torna indispensável a sua ingestão mediante dieta. Os alimentos de origem vegetal podem suprir a maior parte das vitaminas necessárias ao organismo, mas a sua importância advém, principalmente, pelo suprimento de vitamina C. Por serem consumidas in natura, na maioria das vezes, as frutas são melhores fontes que as hortaliças, as 
quais, em grande parte, são submetidas à cocção (Chitarra $\&$ Chitarra 2005). Todos as amostras apresentaram teores de vitamina $\mathrm{C}$ similares entre si e superiores ao determinado pela tabela de composição de frutos $(16,2 \mathrm{mg} 100 \mathrm{~g}$ de polpa $^{-1}$ ) (Taco 2006), variando entre 17,68 e 22,14 mg 100 g de polpa ${ }^{-1}$ (Tab. 3).

Silva et al. (2008) estudando a estabilidade da vitamina $\mathrm{C}$ em três estágios de maturação de cagaita (Eugenia dysenterica DC.), encontraram grandes variações nos teores de vitamina C (entre 20,21 e 27,46 mg/100g de vitamina $\mathrm{C}$ ), contudo, superiores aos encontrados neste estudo para jabuticabas, onde utilizou-se frutos maduros. Segundo os autores, a grande variação entre plantas e, consequentemente, entre frutos produzidos em árvores diversas, podem ter influenciado os resultados, apesar da homogeneização das amostras após coleta dos frutos. Silva et al. (2013) descreveram teores similares $(0,71 \mathrm{~g}$ $100 \mathrm{~g}$ de polpa $^{-1}$ de ácido cítrico) aos obtidos nesse estudo em resíduo proveniente da despolpação da jabuticaba. Já Boari Lima et al. (2008) observaram teores superiores na polpa fresca de jabuticabas Paulista ( $P$. cauliflora) e Sabará (P. jaboticaba), com 1,37 e 1,67 g $100 \mathrm{~g}$ de polpa ${ }^{-1}$ de ácido cítrico, respectivamente) e Dessimoni Pinto et al. (2011) evidenciaram níveis inferiores de acidez para casca de jabuticaba $\left(0,31 \mathrm{~g} \mathrm{mg} 100 \mathrm{~g}_{\text {de polpa }}{ }^{-1}\right.$ de ácido cítrico). Silva et al. (2001), em cagaita (E. dysenterica), e Vera et al. (2005), em pequi (Caryocar brasiliense A. St.Hil.), observaram grandes variações nos caracteres físicos de frutos em estudos de caracterização. O que reforça a sugestão de variabilidade química entre frutos.

A amostra 2, seguida das amostras 6 e 5 apresentaram maior luminosidade ( $\left.\mathrm{L}^{*}\right)$ com $(24,24 ; 24,11$ e 23,63 , respectivamente) (Tab. 4). Para os índices $\mathrm{a}^{*} \mathrm{e} \mathrm{b}^{*} \mathrm{e}$ cromaticidade a amostra 6 foi inferior aos demais, embora não tenha diferido das amostras 3 e 4 para o índice $b^{*}$ (Tab. 4).

Tabela 3. Sólidos solúveis ( ${ }^{\circ}$ Brix), Acidez Titulável (\% ácido citrico), Ratio (sólidos solúveis e acidez titulável) e teor de vitamina C (mg / $100 \mathrm{~g}$ de polpa) de frutos de cinco amostras de jabuticabeira coletados em Porto Alegre.

\begin{tabular}{|c|c|c|c|c|}
\hline $\begin{array}{l}\text { Comparação entre: } \\
\text { Amostras/indivíduos }\end{array}$ & Sólidos Solúveis $\left({ }^{\circ}\right.$ Brix $)$ & $\begin{array}{c}\text { Acidez Titulável } \\
\text { (\% de ácido cítrico) }\end{array}$ & Ratio (SS/AT) & $\begin{array}{c}\text { Teor de Vitamina C } \\
\text { (mg / } 100 \mathrm{~g} \text { de polpa) }\end{array}$ \\
\hline P. peruviana (3) & $11,97 \quad a b$ & $0,74 \quad b c$ & $14,27 \quad \mathrm{c}$ & 18,93 \\
\hline P. peruviana (4) & $11,40 \quad b$ & 0,82 a & $13,62 \mathrm{~cd}$ & 18,10 \\
\hline P. peruviana (6) & 12,75 a & $0,69 \mathrm{c}$ & $17,10 \quad \mathrm{a}$ & 19,63 \\
\hline P. cauliflora (2) & $12,20 \quad a b$ & 0,83 a & $13,12 \quad b$ & 22,14 \\
\hline P. cauliflora (5) & $12,40 \quad \mathrm{a}$ & 0,78 ab & $15,73 \quad \mathrm{~b}$ & 17,68 \\
\hline \multicolumn{5}{|l|}{ Espécies } \\
\hline P. peruviana & 12,04 & $0,84 \quad b$ & 16,09 & 18,88 \\
\hline P. cauliflora & 12,30 & $0,90 \quad \mathrm{a}$ & 15,29 & 19,91 \\
\hline CV \% Amostras & $3,05 \quad * * *$ & $5,25 \quad * * *$ & $7,42 \quad * * *$ & $12,56(\mathrm{~ns})$ \\
\hline CV \% Espécies & $4,67 \quad(\mathrm{~ns})$ & $17,76 \quad * * *$ & $10,89 \quad(\mathrm{~ns})$ & $14,06(\mathrm{~ns})$ \\
\hline
\end{tabular}

Médias seguidas de mesma letra, na coluna, não diferem entre si pelo teste de Duncan ( $p<0,05)$, entre as amostras e LSD (p $<0,001)$ entre as espécies, ns: Variância não significativa.

Tabela 4. Índices de cor ( $\mathrm{L}^{*}, \mathrm{a}^{*}$ e b*), cromaticidade e ângulo hue de frutos de cinco amostras de jabuticabeira coletados em Porto Alegre.

\begin{tabular}{|c|c|c|c|c|c|c|c|c|c|}
\hline \multirow{3}{*}{$\begin{array}{l}\text { Comparação entre: } \\
\text { Amostras/indivíduos } \\
\text { P. peruviana (3) }\end{array}$} & \multicolumn{6}{|c|}{ Índices de cor } & \multirow{2}{*}{\multicolumn{2}{|c|}{ Cromaticidade }} & \multirow{3}{*}{$\begin{array}{c}\text { Ângulo hue } \\
15,07\end{array}$} \\
\hline & \multicolumn{2}{|c|}{$\mathrm{L}^{*}$} & \multicolumn{2}{|c|}{$\mathrm{a}^{*}$} & \multicolumn{2}{|c|}{$\mathrm{b}^{*}$} & & & \\
\hline & 23,01 & $\mathrm{~b}$ & 4,24 & $\mathrm{a}$ & 1,13 & $\mathrm{ab}$ & 4,39 & $\mathrm{a}$ & \\
\hline P. peruviana (4) & 23,04 & $\mathrm{~b}$ & 3,83 & $\mathrm{a}$ & 1,10 & $\mathrm{ab}$ & 3,99 & $\mathrm{a}$ & 15,99 \\
\hline P. peruviana (6) & 24,11 & $\mathrm{a}$ & 2,59 & $\mathrm{~b}$ & 0,88 & $\mathrm{~b}$ & 2,75 & $\mathrm{~b}$ & 18,63 \\
\hline P. cauliflora (2) & 24,24 & $\mathrm{a}$ & 4,24 & $\mathrm{a}$ & 1,23 & $\mathrm{a}$ & 4,42 & $\mathrm{a}$ & 16,18 \\
\hline P. cauliflora (5) & 23,63 & $\mathrm{ab}$ & 3,94 & $\mathrm{a}$ & 1,30 & a & 4,16 & a & 18,29 \\
\hline \multicolumn{10}{|l|}{ Espécies } \\
\hline P. peruviana & 23,39 & $\mathrm{~b}$ & 3,55 & $\mathrm{~b}$ & 1,03 & $\mathrm{~b}$ & 3,71 & & 16,56 \\
\hline P. cauliflora & 23,94 & $\mathrm{a}$ & 4,09 & a & 1,26 & $\mathrm{a}$ & 4,29 & & 17,24 \\
\hline CV \% Amostras & 1,79 & $* * *$ & 10,47 & $* * *$ & 17,16 & $* * *$ & 10,31 & $* * *$ & $15,95(\mathrm{~ns})$ \\
\hline CV \% Espécies & 6,36 & $* *$ & 35,59 & $* *$ & 66,74 & * & 17,39 & (ns) & $16,82(\mathrm{~ns})$ \\
\hline
\end{tabular}

Médias seguidas de mesma letra, na coluna, não diferem entre si pelo teste de Duncan $(p<0,05)$, entre as amostras e LSD (p $<0,001)$ entre as espécies, ns =variância não significativa. 
Não houve diferença entre as amostras para o ângulo hue (Tab. 4). Os valores a* positivos, encontrados em todos os frutos indicam a presença da tonalidade vermelha na epiderme dos frutos. Assim, como os valor b*, próximos de zero indicam a presença de azul, o que determina o escurecimento dos frutos. Os valores de cromaticidade encontrados indicam que os frutos são vermelhos embora a cor não seja pura.

Quando avaliado o desenvolvimento pós-colheita, em todas amostras houveram perdas significativas, com tendência linear para perda de massa fresca (de 100\% na data inicial para cerca de $94 \%$ ao final do período), atingindo redução ao redor de $6 \%$ no final do período de armazenamento (Fig. 1). Contudo essas perdas não causaram severos murchamentos e/ou enrugamentos, capazes de comprometer a qualidade visual dos frutos. Corroborando assim, com o já observado por Vieites et al. (2011), em estudo com diferentes temperaturas de armazenamento de frutos de jabuticaba (P. jaboticaba), durante 30 dias, onde foi observado $2 \%$ de perda de massa, sem sinais de murchamento e/ou enrugamento. Cabe destacar que perdas de massa fresca na faixa de 5 a $10 \%$ são toleradas para produtos in natura, e podem não provocar o aparecimento de murcha e enrugamento na superfície (Finger \& Vieira 2002).

Houve incremento, com tendência linear para as todas amostras quanto ao teor de vitamina $\mathrm{C}$ ao longo do período de armazenamento (Fig. 2), provavelmente devido à perda de massa dos frutos durante o armazenamento (Fig. 1). Somente a amostra 6 não apresentou mudanças significativas no teor de vitamina $\mathrm{C}$ durante o armazenamento. Já em polpa de fruta de Cagaita ( $E$. dysenterica), armazenada a $-18^{\circ} \mathrm{C}$ durante quatro meses, Silva et al. (2008) observaram que a concentração de vitamina $\mathrm{C}$ reduziu-se, gradativamente, com diminuição de, aproximadamente, $30 \%$, no primeiro mês, e $50 \%$, no terceiro mês. Provavelmente devido à oxidação química do ácido ascórbico e/ou degradação térmica como consequência do congelamento da polpa.

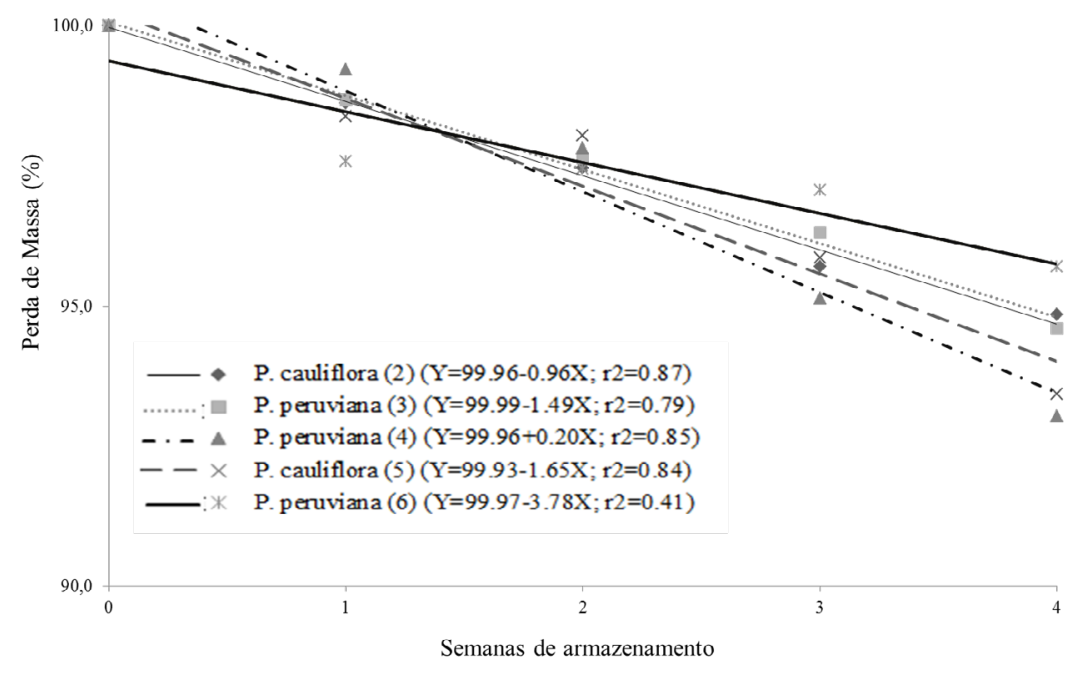

Fig. 1. Percentual de perda de massa de frutos de cinco amostras de jabuticabeira, armazenadas durante quatro semanas sob-refrigeração $\left(\approx 2^{\circ} \mathrm{C}\right)$.

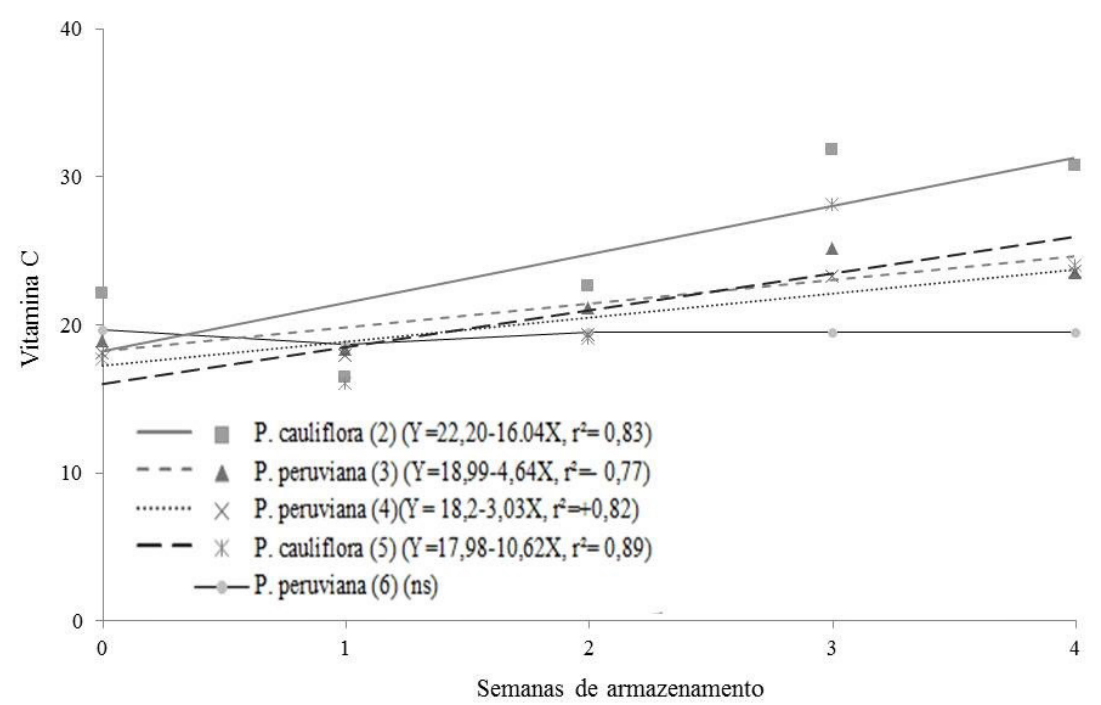

Fig. 2. Teor de vitamina $\mathrm{C}$ de frutos de cinco amostras de jabuticabeira, armazenadas durante quatro semanas sob-refrigeração $\left(\approx 2^{\circ} \mathrm{C}\right)$. 
Normalmente, os teores de açúcares aumentam com o amadurecimento das frutas por meio da degradação de polissacarídeos, quando há conversão de amido em açúcares solúveis. Outro fator que contribui para o aumento dos sólidos solúveis é a perda de massa fresca, o que faz com que os sólidos fiquem mais concentrados no suco. $\mathrm{O}$ teor de sólidos solúveis pode diminuir após um período prolongado de armazenamento, caso o consumo de açúcares como substrato no processo respiratório seja superior aos processos de degradação de polissacarídeos (Siqueira 2012).

De forma geral, as amostras apresentaram decréscimos no teor de sólidos solúveis ao longo do período de armazenamento, o que pode ser devido á perda de massa (Fig. 1) que promove a concentração de açucares na polpa do fruto. As amostras 2 e 3 não apresentaram regressões significativas para o conteúdo de sólidos solúveis das jabuticabas (Fig. 3), contudo as amostras 4 e 6 apresentaram tendência quadrática, enquanto a amostra 5 , tendência linear. Vieites et al. (2011) testando diferentes temperaturas de armazenamento, observaram acréscimo inicial no teor de sólidos solúveis, seguido de progressivos decréscimos, justificando tal comportamento como consequência do metabolismo respiratório climatérico das jabuticabas (P. jaboticaba). Sendo ainda este comportamento relativo ao estádio de maturação dos frutos.

Foram observados incrementos na acidez titulável em todas amostras analisadas ao longo do período de armazenamento, exceto para a amostra 6 (Fig. 4), sendo este comportamento justificado pelo avanço do processo de maturação do fruto, visto que os ácidos orgânicos tendem a diminuir durante o amadurecimento dos frutos em virtude de sua utilização como substrato respiratório (Calegano et al. 2002).

Considerando os resultados obtidos, Plinia cauliflora e $P$. peruviana apresentam características físicoquímicas similares, podendo os frutos maduros serem armazenadas por 28 dias sob-refrigeração $\left(\approx 2^{\circ} \mathrm{C}\right)$, sem perdas significativas da sua qualidade (aspectos físicoquímicos e visuais).

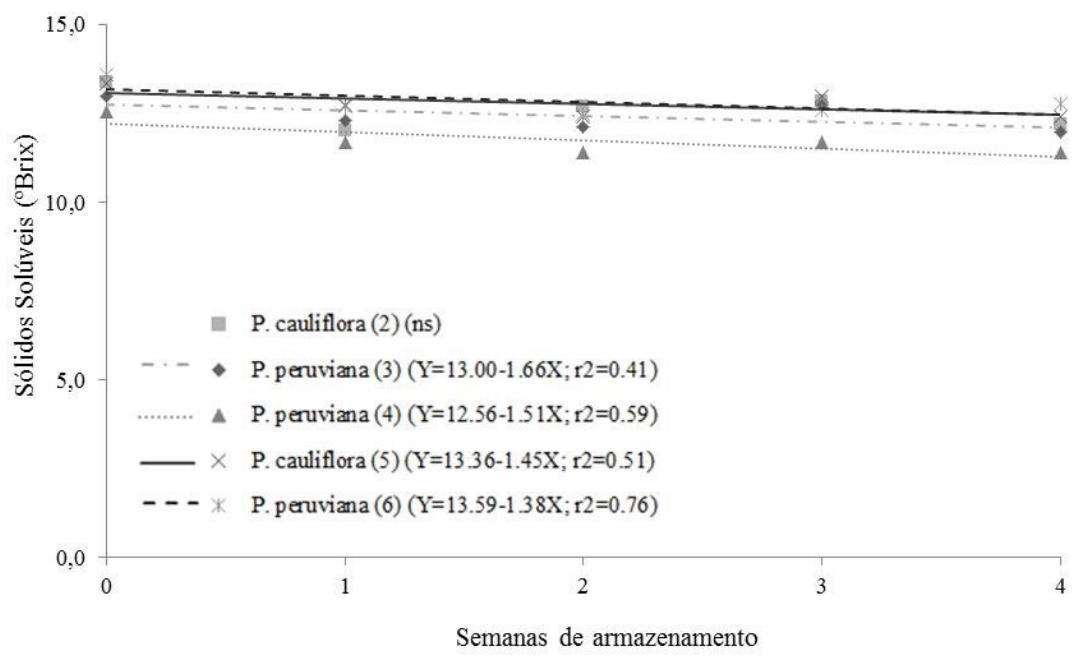

Fig. 3. Teor de sólidos solúveis de frutos de cinco amostras de jabuticabeira, armazenadas durante quatro semanas sob-refrigeração $\left(\approx 2^{\circ} \mathrm{C}\right)$.

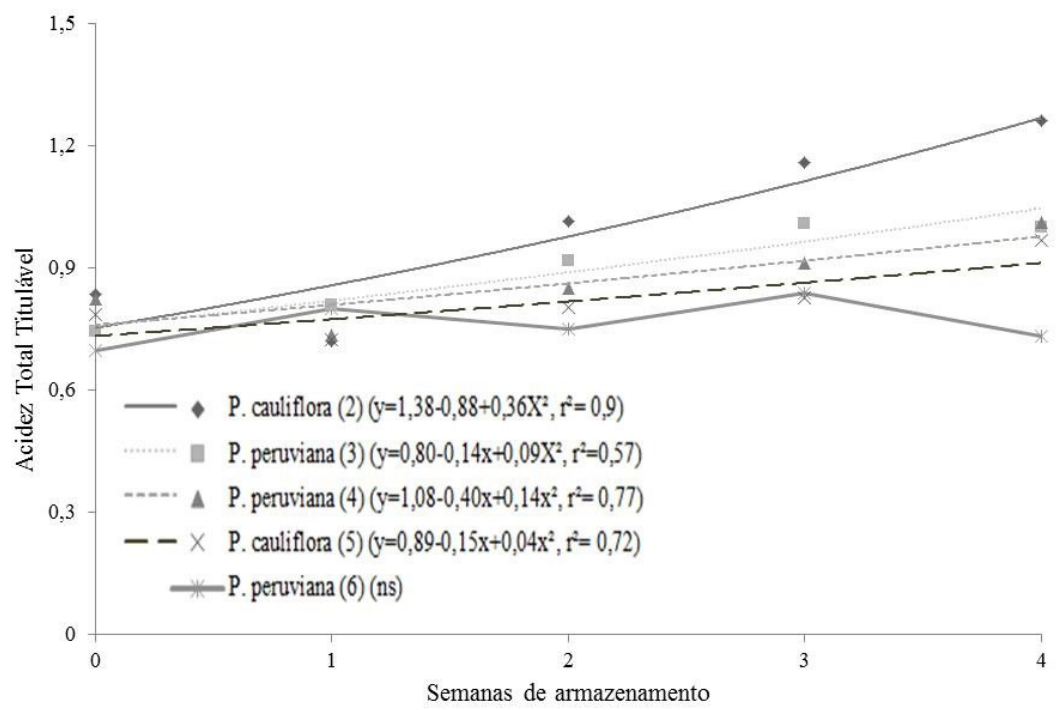

Fig. 4. Acidez titulável de frutos de cinco amostras de jabuticabeira, armazenadas durante quatro semanas sob-refrigeração $\left(\approx 2^{\circ} \mathrm{C}\right)$. 


\section{AGRADECIMENTOS}

Ao Eng. Agr. André Bordignon pela disponibilização do pomar para coleta das amostras de jabuticabas. Ao Eng. Agr. Ernani Pezzi pelo auxílio nas análises laboratoriais.

Ao CNPq pelo auxilio financeiro.

$\mathrm{O}$ presente trabalho foi realizado com apoio da Coordenação de Aperfeiçoamento de Pessoal de Nível Superior - Brasil (CAPES) - Código de Financiamento 001.

\section{REFERÊNCIAS}

Association of official analytical chemists - AOAC. 1997. Official methods of analysis of the AOAC. Washington, 2: 16-17.

Calegano, J.M.; Pezzi, E. \& Bender, R.J. 2002. Utilização de atmosfera modificada na conservação de morangos pós-colheita. Pesquisa Agropecuária Brasileira, 37(8):1049-1055.

Chitarra, M. I. F. \& Chitarra, A. B. 2005. Pós-colheita de frutos e hortaliças: fisiologia e manuseio. Universidade Federal de Lavras, Lavras. 785 p.

Boari Lima, A.J., Correâ, A.D., Alves, A.P.C., Abreu, C.M.P. \& Dantas-Barros, A.M. 2008. Caracterização química do fruto jabuticaba (Myrciaria cauliflora Berg) e de suas frações. Archivos Latinoamericanos de Nutrición, 58(4):416-421.

Danner, M.A., Citadin, I., Sasso, S.A.Z., Sachet, \& M.R., Malagi, G. 2011. Modo de reprodução e viabilidade de pólen de três espécies de jabuticabeira. Revista Brasileira de Fruticultura, Jaboticabal. 33(2):345-352.

Dessimoni-Pinto, N.A.V., Moreira, W.A., Cardoso, L.M., \&Pantoja, L.A. 2011. Jaboticaba peel for jelly preparation: an alternative technology. Ciência e Tecnologia de Alimentos, 31(4):864-869.

Finger, F.L. \& Vieira, G. 2002. Controle da perda pós-colheita de água em produtos hortícolas. Universidade Federal de Viçosa, Viçosa. 29 p.

Flora do Brasil 2020 em construção. Jardim Botânico do Rio de Janeiro. Disponível em: <http://floradobrasil.jbrj.gov.br/ > . Acesso em: 03 Set. 2019

Instituto Brasileiro de Geografia e Estatistica - IBGE. 2017. Censo Agropecuário 2017. Resultados Preliminares. Brasil, Disponível em: https://censos.ibge.gov.br/agro/2017/templates/censo_agro/ resultadosagro/agricultura.html localidade $=0 \&$ tema $=76309$. Acesso em: 03 set. 2019.

Kluge, R.A., Nachtigal, J.C., Fachinello, J.C. \& Bilhalva, A.B. 2002. Fisiologia e manejo pós-colheita de frutas de clima temperado. Livraria e Editora Rural, Campinas. 214 p.

Martins, R. 2007. Dossiê técnico: doce em pasta e em calda. Rede de Tecnologia do Rio de Janeiro (REDETEC), Rio de Janeiro. 39 p.

Negreiros, J.R.S. Neto, S.E.A., Álvares, V.S., Lima, V.A., \& Oliveira, T.K. 2008. Caracterização de frutos de progênies de meios-irmãos de maracujazeiro-amarelo em Rio Branco - Acre. Revista Brasileira de Fruticultura 30(2):431-437.

Oliveira, A.L., Brunini, M.A., Salandini, C.A.R., \& Bazzo, F.R. 2003. Caracterização tecnológica de jabuticabas 'Sabará' provenientes de diferentes regiões de cultivo. Revista Brasileira de Fruticultura 25(3):393-400.

Plageman, I., Krings, I., Berger, R. G. \& Maróstica Júnior, M. R. 2012. Volatile constituents of jabuticaba (Myrciaria jaboticaba - Vell. O. Berg) fruits. Journal of Essential Oil Research, 24(1):45-51.

Ronchi, H.S. 2017. Potencial alimentício e medicinal das espécies nativas da área de proteção ambiental - APA Corumbataí, Botucatu e Tejupá - perímetro Botucatu. Dissertação 111 f. Universidade Estadual Paulista, Botucatu.

Sasso, S.A.Z, Citadin, I., Danner, M.A. 2010. Propagação de jabuticabeira por enxertia e alporquia. Revista Brasileira de Fruticultura 32(2):571576.

Silva, R.S.M., Chaves, L.J. \& Naves, R.,V. 2001. Caracterização de frutos e árvores de cagaita (Eugenia dysenterica DC.) no sudeste do Estado de Goiás, Brasil. Revista Brasileira de Fruticultura 23(2):330-334.

Silva, M.R., Santos Júnior, R.T.O.J., \& Ferreira, C.C.C. 2008. Estabilidade da vitamina c em cagaita in natura e durante a estocagem da polpa e refresco. Pesquisa Agropecuária Tropical 38(1):53-58.

Silva, P.I., Stringheta, P.C., Teófilo, R F. \& Oliveira, I R N. 2013. Parameter optimization for spray-drying microencapsulation of jaboticaba (Myrciaria jaboticaba) peel extracts using simultaneous analysis of responses. Journal of Food Engineering 117:538-554.

Silva, E.E.A.N., Soares, D.S.B., Oliveira, T.M., Pinto, E.G., Nascimento, W.V. \& Souza, A.R. M. 2017. Aplicação de cobertura de quitosana em jabuticabas. Revista Agrarian 10(38):363-369.

Silveira, N.S.S., Michereff S. J., Silva, I.L.S. \& Oliveira A. M. 2005. Doenças fúngicas pós-colheita em frutas tropicais: patogênese e controle. Caatinga 18(4):283-299.

Siqueira, A.P.O. 2012. Uso de coberturas comestíveis na conservação pós-colheita de goiaba e maracujá-azedo, $91 \mathrm{f}$. Universidade Estadual do Norte Fluminense Darcy Ribeiro, Campo dos Goytacazes.

Tabela Brasileira de Composição de Alimentos - TACO. 2006. Unicamp, Campinas. $113 \mathrm{p}$.

Vera, R., Naves, R.V., Nascimento, J.L., Chaves, L.J., Leandro, W.M. \& Souza, E.R.B. 2005. Caracterização física de frutos do pequizeiro (Caryocar brasiliense Camb.) no Estado de Goiás. Pesquisa Agropecuária Tropical 35(2):71-79.

Vieites, R.L., Daiuto, E.R., Moraes, M.R., Neves, L.C. \& Carvalho, L.R. 2011. Caracterização Físico-química, Bioquímica e Funcional da Jabuticaba armazenada sob diferentes temperaturas, Revista Brasileira de Fruticultura 33(2):362-375.

Zerbielli, L., Nienow, A.A., Dalacorte, L., Jacobs, R. \& Daronch, T. 2016. Diversidade físico-química dos frutos de jabuticabeiras em um sítio de ocorrência natural. Revista Brasileira Fruticultura 38(1):107-116. 